\title{
Driving force for a nonequilibrium phase transition in three-dimensional complex plasmas
}

\author{
D. I. Zhukhovitskii* \\ Joint Institute of High Temperatures, Russian Academy of Sciences, Izhorskaya 13, Bd. 2, 125412 Moscow, Russia
}

(Dated: July 6, 2021)

\begin{abstract}
An example of the non-equilibrium phase transition is the formation of lanes when one kind of particles is driven against the other. According to experimental observation, lane formation in binary complex plasmas occurs when the smaller particles are driven through the stationary dust cloud of the larger particles. We calculate the driving force acting on a probe particle that finds itself in a quiescent cloud of particles in complex plasma of the low-pressure radio frequency discharge under microgravity conditions. It is shown that the nonzero driving force is a result of the dependence of the ion mean free path on the particle number density. If this effect is properly included in the model of similar complex plasmas then one arrives at the driving force that changes its sign at the point where the probe and the dust particles have equal radii. If the probe is smaller than the dust particle then the driving force is directed toward the discharge center and vice versa, in accordance with experiment. Obtained results can serve as the ansatz for future investigation of the lane formation in complex plasmas.
\end{abstract}

PACS numbers: 52.27.Lw, 82.70.-y, 05.65.+b

\section{INTRODUCTION}

Complex plasmas are low-temperature plasmas including dust particles, typically in the micrometer range. These are dusty plasmas, which are specially prepared to study fundamental processes in the strong coupling regime on the most fundamental level when the observation of individual motion of particles and their interactions is possible. Under microgravity conditions realized either in parabolic flights ${ }^{1-5}$ or onboard the International Space Station (ISS), ${ }^{1,6-11}$ such relatively weak forces as the ion drag force and the interparticle interactions become important and often determine the motion and structure formation in complex plasma. Since the mobility of electrons is much greater than that of ions, particles acquire a significant negative electric charge. This leads to formation of a strongly coupled plasma, ${ }^{12-19}$ in which large volumes of almost homogeneous three-dimensional complex plasma can be observed.

This strongly coupled open system can exhibit a number of nonequilibrium phase transitions. Among them, one of the most vivid effects is the lane formation when two kinds of particles are driven against each other. If the driving forces are strong enough like particles form "stream lines" and move collectively in lanes, which show an anisotropic structural order accompanied by a considerable enhancement of the particle mobility. Examples of this phenomenon include driven bi-layer systems and two-species lattice gases ${ }^{20}$ granular mixtures,${ }^{21}$ molecular ions, ${ }^{22}$ highly populated pedestrian zones, ${ }^{23}$ and driven colloidal mixtures. ${ }^{24-31}$ Lane formation in threedimensional complex plasma of a dust cloud in the radio frequency low-pressure gas discharge was observed in Refs. 32 and 33.

Lane formation in complex plasmas is a special issue for two reasons. First, only one species of the dust particles is driven while the second one is quiescent. Note that in this case, the only difference between the two species is the particle radius. Obviously, this peculiarity is not of principal nature. Second, the driving force is not an external one but it is a sum of the internal forces acting on the particle in plasma. In most cases, these forces are the oppositely directed electric and ion drag forces, which cannot be directly controlled. Moreover, while, e.g., the driving force in colloidal mixtures is treated as a preassigned parameter, it is not known for complex plasmas. Thus, calculation of the driving force becomes a separate problem, which is addressed in this work.

The force acting on an isolated dust particle is wellknown. ${ }^{12}$ However, this approach is inappropriate for the strongly coupled dust cloud where the interparticle interactions are quite essential. In the recent studies, ${ }^{34,35}$ the electric and ion drag forces are calculated assuming that the Coulomb potentials of neighboring particles overlap. This provides an interpretation for the particle number density in a dust cloud and determines a relationship between the number densities of all plasma charge carriers and the particle charge. We will term the force acting on an individual probe particle of the radius $a_{p}$ that finds itself in a dust cloud of particles of the radius $a$ the driving force. Surprisingly, calculation of this force based on the model of similar complex plasmas $(\mathrm{SP})^{35}$ results in the force vanishing.

In this work, we will show that the nonzero driving force is a net result of the fact that the ion mean free path with respect to the collisions against neutrals can be comparable with the interparticle distance, which was not taken into account in Ref. 35. If we calculate the total mean free path with due regard for the ion scattering on the dust particles then the ionization equation of state (IEOS) is modified so that the driving force does not vanish. In this way, we derive the modified model of similar complex plasmas (MSP). The calculation using MSP shows that if $a_{p}>a$, the larger subsonic particle 
that we term the probe moves through the dust cloud along the ion flux toward the outer boundary of a dust cloud, in correspondence with the experiments. For the larger supersonic particle, the estimations show that due to the absence of a spherical cavity around the moving particle the ion drag force is much weaker than in the case of a subsonic particle. Hence, the driving force is not much different from the electric force and the probe velocity is directed against the ion drag force toward the discharge center. The developed approach can be used as an ansatz for development of the theory of lane formation in complex plasmas.

The paper is organized as follows. In Sec. II, the IEOS for the stationary dust cloud obtained in our previous studies is generalized to include the effect of finite ion mean free path affected by the collisions against dust particles. In Sec. III, we explore the modified IEOS and derive the pressure of the dust particle subsystem necessary for the calculation of driving force and check the effect of IEOS modification on the velocity of dust acoustic waves. The driving force is calculated in Sec. IV, and the resulting larger particle velocity is estimated and compared with experiments in Sec. V. The results of this study are summarized in Sec. VI, and the parameters of a cavity behind the supersonic probe are estimated in the Appendix A.

\section{MODIFIED IONIZATION EQUATION OF STATE}

Consider a stationary dust cloud in the low-pressure gas discharge. Here and in Sec. III, we imply that the particles forming a cloud have the same radius. Under microgravity conditions, a dust particle is subject to three basic forces, namely, the electric driving force, the ion drag force arising from scattering of the streaming ions on dust particles, and the neutral drag force (friction force) due to collisions of the atoms against the moving particles. For a stationary cloud, the latter force vanishes. Note that in a strongly coupled system, the correlation energy originating from particle ordering results in the difference between the volume-averaged electric field and the electric field at the point of particle location. This effect can be included if we introduce the dust pressure. The effect of this pressure on the force balance equation in a stationary plasma is in most cases negligible; however, it is responsible for propagation of a perturbation. ${ }^{35}$ The electric field driving force $\mathbf{f}_{e}$ and the ion drag force $\mathbf{f}_{i d}$ acting on unit volume can be written in the form

$$
\mathbf{f}_{e}=-Z e n_{d} \mathbf{E}=-\frac{a T_{e}}{e} \Phi n_{d} \mathbf{E},
$$

where $Z$ is the dust particle charge in units of the electron charge, $e$ is the elementary electric charge, $n_{d}$ is the particle number density, $a$ is the particle radius, $T_{e}$ is the electron temperature, $\Phi=Z e^{2} / a T_{e}$ is the dimensionless potential of a dust particle, $\mathbf{E}=\left(T_{e} / e\right) \boldsymbol{\nabla} \ln n_{e}$ is the electric field strength, $n_{e}$ is the electron number density, and

$$
\mathbf{f}_{i d}=\frac{3}{8}\left(\frac{4 \pi}{3}\right)^{1 / 3} \beta^{1 / 3} n_{d}^{1 / 3} n_{i} \lambda e \mathbf{E},
$$

where $\lambda$ is the ion mean free path, $n_{i}$ is the ion number density, and $\beta$ is a dimensionless free parameter. Equation (2) implies that the cross section of momentum transfer from the ions to dust particles is $\sigma_{\text {eff }}=$ $(\pi / 2) \beta^{1 / 3} r_{d}^{2}$, where $r_{d}=\left(3 / 4 \pi n_{d}\right)^{1 / 3}$ is the dust particle Wigner-Seitz cell radius. The coefficient $\beta$ depends on $\Phi, r_{d}$, and the ion temperature $T_{i}$ (in Refs. 34 and $35, \sigma_{\text {eff }}$ was estimated using the particle charge screening length in the Wigner-Seitz cell $\simeq 0.45 r_{d}$ that corresponds to $\beta=1$ ).

The force balance equation reads $\mathbf{f}_{e}+\mathbf{f}_{i d}=0$ or

$$
\frac{\pi}{2} \beta^{1 / 3} r_{d}^{2} n_{i} \lambda=\frac{a T_{e}}{e^{2}} \Phi
$$

The ion mean free path $\lambda$ appearing in Eq. (3) is defined by the collisions both with the gas atoms and with the dust particles. In contrast to Refs. 34 and 35, we take this into account and calculate $\lambda$ as

$$
\lambda^{-1}=\lambda_{a}^{-1}+\sigma_{\mathrm{eff}} n_{d}=\lambda_{a}^{-1}\left(1+\frac{3}{8} n_{d}^{* 1 / 3}\right),
$$

where $\lambda_{a}$ is the ion mean free path with respect to the collisions against gas atoms (in a gas discharge without particles) and $n_{d}^{*}=(4 \pi / 3) \beta n_{d} \lambda_{a}^{3}$ is the dimensionless particle number density.

The combination of Eqs. (3) and (4) with the equation for particle potential that follows from the orbital motion limited (OML) approximation ${ }^{36,37}$ at $T_{e} / T_{i} \gg 1$

$$
\theta \Phi e^{\Phi}=\frac{n_{e}}{n_{i}}
$$

where $\theta=\sqrt{T_{e} m_{e} / T_{i} m_{i}}, m_{e}$ and $m_{i}$ is the electron and ion mass, respectively, $n_{e}$ is the electron number density and the local quasineutrality condition

$$
n_{i}=\frac{a T_{e}}{e^{2}} \Phi n_{d}+n_{e},
$$

yield the modified IEOS

$$
\theta \Phi e^{\Phi}+\frac{3}{8}\left(\frac{\pi \tilde{n}_{i}^{*}}{2 \Phi}\right)^{1 / 2}=1,
$$

where

$$
\tilde{n}_{i}^{* 1 / 3}=\frac{n_{i}^{* 1 / 3}}{1+\frac{3}{8}\left(\frac{4 \pi}{3} \frac{\gamma n_{i}^{*}}{\Phi}\right)^{1 / 3}},
$$

$n_{i}^{*}=\beta e^{2} \lambda_{a}^{3} n_{i} / a T_{e}$ is the dimensionless ion number density, and $\gamma(\Phi)=1-\theta \Phi e^{\Phi}$. If we replace $\tilde{n}_{i}^{*}$ by $n_{i}^{*}$ in Eq. (7) it would coincide with Eq. (12) in Ref. 35, i.e., 


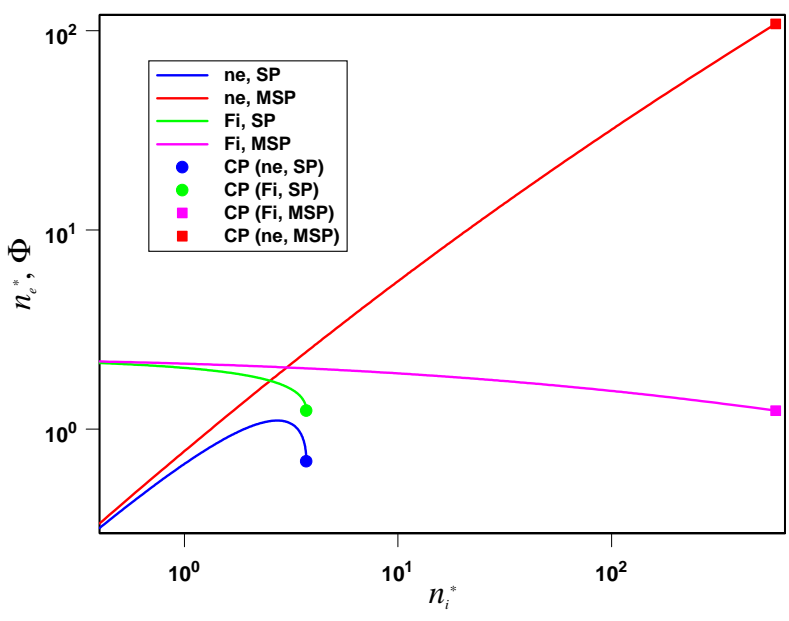

FIG. 1. Dimensionless electron number density $n_{e}^{*}$ from SP (blue line) and MSP (red line) and the particle potential $\Phi$ from SP (green line) and MSP (magenta line) as a function of the dimensionless ion number density $n_{i}^{*}$ for $\theta=0.0431$. Solid dots indicate positions of the critical points in SP (circles) and MSP (squares).

the initial and modified IEOS have the same form. From Eqs. (7) and (8), the ion number density can be represented as an explicit function of $\Phi$

$$
n_{i}^{*}=\frac{128}{9 \pi} \frac{\Phi \gamma^{2}}{(1-\gamma)^{3}}
$$

Given $\Phi$ or $n_{i}^{*}$, one can calculate the particle number density

$$
n_{d}^{*}=\frac{4 \pi}{3} \frac{\gamma n_{i}^{*}}{\Phi}
$$

and the dimensionless electron number density $n_{e}^{*}=$ $\beta e^{2} \lambda_{a}^{3} n_{e} / a T_{e}$,

$$
n_{e}^{*}=n_{i}^{*} \theta \Phi e^{\Phi}=\frac{2}{\pi} \Phi n_{d}^{* 2 / 3} .
$$

Figures 1 and 2 compare the plasma parameters calculated using Eq. (12) in Ref. 35 (SP) and Eq. (9) (MSP). It is seen that at low number densities of the charged components, the calculation results almost coincide, while the maximum $n_{i}^{*}$ that satisfies the modified IEOS (9) is significantly greater. The same is true for $n_{e}^{*}$ and $n_{d}^{*}$. A remarkable difference is observed between the critical points in SP and MSP (Fig. 1). By definition, at the critical point, $n_{i}^{*}$ reaches maximum and the cloud is stable, $d p^{*} / d n_{d}^{*}>0$, where $p^{*}$ is the dimensionless pressure (see Sec. III). For SP, $d n_{i}^{*} / d n_{e}^{*}=d n_{i}^{*} / d \Phi=0$ at the critical point while for MSP, these derivatives do not vanish. The critical $n_{i}^{*}$ is much greater for MSP than for SP. Obviously, such number densities are not realistic because the rate of ion-electron recombination on the particle surface would be too high to sustain the discharge. At the

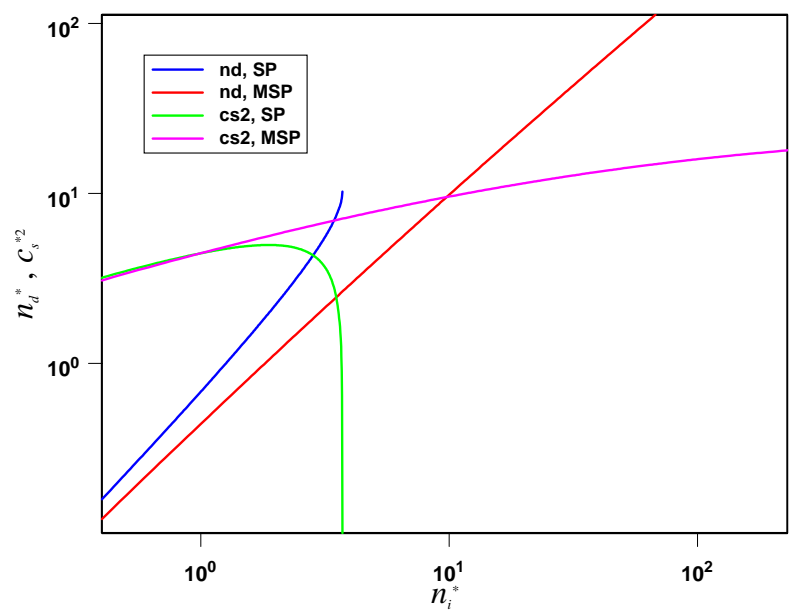

FIG. 2. Dimensionless particle number density $n_{d}^{*}$ from SP (blue line) and MSP (red line) and the squared sound velocity $c_{s}^{2}$ from SP (green line) and MSP (magenta line) as a function of the dimensionless ion number density $n_{i}^{*}, \theta=0.0431$.

same time, in contrast to SP, ${ }^{34}$ MSP does account for high particle number densities in the vicinity of the void boundary. Recall that the void is the dust particle free region in the center of the discharge. ${ }^{12}$

\section{DUST PARTICLE PRESSURE AND THE SOUND VELOCITY}

The dust particle pressure is defined $\mathrm{as}^{34}$

$$
p=\frac{Z^{2} e^{2}}{8 \pi r_{d}^{4}}
$$

One can introduce the dimensionless pressure

$$
p^{*}=\frac{8 \pi e^{2} \lambda_{a}^{4} \beta^{4 / 3}}{a^{2} T_{e}^{2}} p=\Phi^{2} n_{d}^{* 4 / 3} .
$$

The dependence $p^{*}\left(n_{d}^{*}\right)$ is shown in Fig. 3. As is seen, for this quantity, the difference between SP and MSP is insignificant.

The IEOS for the variables $n_{d}^{*}$, $\Phi$ follows straightforwardly from (9) and (10)

$$
n_{d}^{* 1 / 3}=\frac{8 \gamma}{3(1-\gamma)}
$$

We introduce the dimensionless velocity of dust acoustic waves (sound velocity)

$$
c_{s}^{*}=\frac{c_{s} e\left(6 M_{d} \lambda_{a}\right)^{1 / 2} \beta^{1 / 6}}{a T_{e}},
$$

where $c_{s}$ is the sound velocity and $M_{d}$ is the mass of a dust particle. Since $c_{s}^{2}=M_{d}^{-1}\left(d p / d n_{d}\right)$, we obtain from 


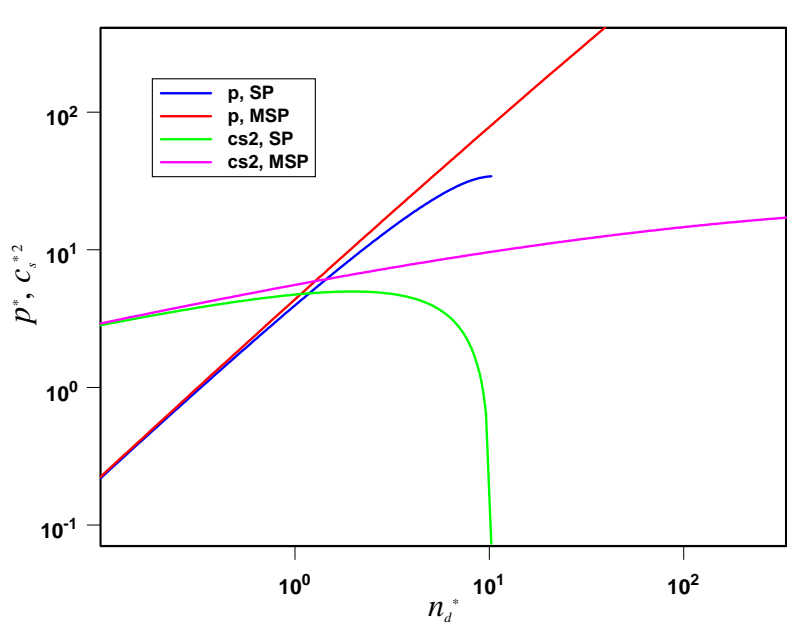

FIG. 3. Dimensionless dust particle pressure $p^{*}$ from SP (blue line) and MSP (red line) and the squared sound velocity $c_{s}^{2}$ from SP (green line) and MSP (magenta line) as a function of the dimensionless particle number density $n_{d}^{*}, \theta=0.0431$.

Eqs. (13)-(15)

$$
c_{s}^{* 2}=\frac{d p^{*}}{d n_{d}^{*}}=\frac{4}{3} \Phi^{2} n_{d}^{* 1 / 3}\left[1-\frac{\gamma}{2(1+\Phi)}\right] .
$$

Eq. (16) differs from corresponding Eq. (35) in Ref. 35. Although according to the modified IEOS (9) the dust pressure is sensitive to $n_{d}^{*}$, the sound velocity is not much different from that obtained in Ref. 35 (Figs. 2 and 3) and, therefore, from the experimental results. Likewise, it is almost independent on $n_{i}$ and $n_{e}$, i.e., on the position in the dust cloud. In contrast to Ref. 35, where the calculated $c_{s}$ vanishes at the critical point, such behavior extends to considerably higher $n_{d}^{*}$, so that this range covers the particle number densities typical for the void boundary. The fact that the number density variation has almost no effect on the sound velocity has been confirmed experimentally (see references in Ref. 35).

\section{CALCULATION OF THE DRIVING FORCE}

In this Section, we will treat a probe particle with the radius $a_{p}$ in the cloud of particles with the radius $a$. Such particles can appear in the discharge sporadically or they can be injected in plasma purposely. Our objective will be the calculation of the sum of the ion drag and electric force acting on this probe particle, which is the driving force. We will term the Wigner-Seitz cell of a probe "cavity." If $R_{p} \gg a$, the cavity is the dust particle free region around a probe. The particles are displaced from the cavity due to the Coulomb repulsion between the probe and particles rather than to the ion drag force as in the case of the void. The radius of a cavity around the probe $R_{p}$ is obtained from the minimization of the work of its formation $(4 \pi / 3) p R_{p}^{3}+Z_{p}^{2} e^{2} / 2 R_{p}$, where $Z_{p}$ is the charge of a probe particle in units of the electron charge, ${ }^{34}$

$$
R_{p}^{2}=\frac{Z_{p} e}{\sqrt{8 \pi p}}=\frac{a_{p}}{a} \frac{\lambda_{a}^{2} \beta^{2 / 3}}{n_{d}^{* 2 / 3}}
$$

and we used (13). It is remarkable that the cavity radius as a function of the dimensional particle number density

$$
R_{p}=r_{d} \sqrt{\frac{a_{p}}{a}}
$$

is independent of $\beta$.

We will assume that at the boundary of a cavity around the probe particle, the electron and ion number densities coincide with $n_{e}$ and $n_{i}$, respectively, i.e., the potentials of dust and probe particles coincide. Hence, $\Phi=$ $Z_{p} e^{2} / a_{p} T_{e}$. If we define the direction of a coordinate axis $Y$ apart from the void center as a positive direction then the electric force acting on the probe is $F_{e p}=a_{p} T_{e} \Phi E / e$, where $E=\left(T_{e} / e\right)\left(d \ln n_{e} / d y\right), y$ is the coordinate, and the ion drag force is $F_{i p}=-(\pi / 2) \beta^{1 / 3} R_{p}^{2} n_{i} \lambda_{p} e E$, where

$$
\lambda_{p}=\frac{\lambda_{a}}{1+\frac{3}{8} \frac{\lambda_{a}}{R_{p}}}=\frac{\lambda_{a}}{1+\frac{\gamma}{1-\gamma} \sqrt{\frac{a}{a_{p}}}}
$$

is the ion mean free path in a cloud with the particle number density $(3 / 4 \pi) R_{p}^{-3}$, and we used (18). With due regard for (10), the ratio of the absolute values $\kappa=-F_{e p} / F_{i p}$ is given by the following equation,

$$
\kappa=1+\left(\sqrt{\frac{a}{a_{p}}}-1\right) \gamma
$$

It is noteworthy that $\kappa$ is independent of $\beta$. Thus, the sought driving force $F_{\mathrm{drv}}=F_{e p}+F_{i p}$ is

$$
F_{\mathrm{drv}}=\frac{a_{p} \Phi T_{e}^{2}}{e^{2}}\left(1-\frac{1}{\kappa}\right) \frac{d \ln n_{e}}{d y} .
$$

If $\left|a_{p}-a\right| / a_{p} \ll 1$ then (21) is reduced to

$$
F_{\mathrm{drv}}=\frac{\gamma \Phi T_{e}^{2}\left(a-a_{p}\right)}{2 e^{2}} \frac{d \ln n_{e}}{d y} .
$$

One can substitute $d \ln n_{e} / d y$ in (21) and (22) by $d \ln n_{d} / d y$ using (11). Since

$$
\frac{d n_{e}^{*}}{d n_{d}^{*}}=\frac{4 \Phi}{3 \pi n_{d}^{* 1 / 3}}-\frac{\Phi(1-\gamma)}{4 \pi(1+\Phi)},
$$

we obtain

$$
\frac{d \ln n_{e}}{d y}=\left[\frac{2}{3}-\frac{n_{d}^{* 1 / 3}(1-\gamma)}{8(1+\Phi)}\right] \frac{d \ln n_{d}}{d y} .
$$


It follows from (20)-(22) that at $a_{p}=a, F_{\mathrm{drv}}=0$, i.e., a homogeneous cloud is stationary, as it must. For $a_{p}>$ $a, \kappa<1$ and $F_{\mathrm{drv}}>0$ if $d \ln n_{e} / d y<0$. Thus, a large subsonic probe moves from the void center toward the outer boundary of a cloud. Such conditions are typical for the experiment, ${ }^{38}$ where an outward motion of a large probe particle was registered. On the contrary, for $a_{p}<$ $a, \kappa>1$ and $F_{\text {drv }}<0$, i.e., a small subsonic probe must move toward the void center. This effect was observed experimentally in Refs. 32 and 33.

Thus, the driving force acting on a probe particle originates from the dependence of the ion mean free path on the particle number density (4). Disregarding this dependence vanishes $F_{\text {drv }}$. In fact, $\lambda \rightarrow \lambda_{a}$ if $n_{d} \rightarrow 0$, which means that $n_{e} \rightarrow n_{i}$. According to (5) in this case, $\gamma \rightarrow 0$ and $F_{\mathrm{drv}} \rightarrow 0$ as it follows from (20) and (21). The result $F_{\text {drv }} \equiv 0$ can be directly obtained using IEOS Eq. (12) in Ref. 35, which is a limit case of Eqs. (7) and (8) for $\gamma \ll 1$.

\section{VELOCITY OF THE PROBE MOTION}

The Newtonian equation that governs the probe motion is

$$
\dot{u}+\nu_{p} u=\frac{F_{\mathrm{drv}}}{M_{p}},
$$

where $u$ is the probe velocity, and

$$
\nu_{p}=\frac{8 \sqrt{2 \pi}}{3} \frac{\delta m_{n} n_{n} v_{T} a_{p}^{2}}{M_{p}}\left(1+\frac{1}{2} \sqrt{\frac{a}{a_{p}}}\right)
$$

is the friction coefficient for a probe, $\delta \simeq 1.44$ is the accommodation coefficient ${ }^{39} m_{n}$ is the mass of a gas molecule; $n_{n}$ and $v_{T}=\left(T_{n} / m_{n}\right)^{1 / 2}$ are the number density and thermal velocity of the gas molecules, respectively, $T_{n}=300 \mathrm{~K}$ is the temperature of a gas; $M_{p}=(4 \pi / 3) \rho_{p} a_{p}^{3}$ and $\rho_{p}$ are the probe particle mass and its material density, respectively. Equation (26) allows for the enhancement of neutral drag force due to the dissipation in the fluid of dust particles surrounding the probe. It was shown in Ref. 40 that this effect leads to an additional factor $1+\left(R_{p} / r_{d}\right)^{3}\left(a / a_{p}\right)^{2}$ in the expression for $\nu_{p}$. With due regard for Eq. (18), we can rewrite this factor in the form that appears in (26). Note that if $a_{p}<a$, Eq. (26) is invalid and it can only be regarded as an extrapolation. Since an appropriate estimation of the friction force for this case is absent, we will use (26) to plot Fig. 4. However, it is noteworthy that the effect of scattering of a smaller probe on larger particles forming the cloud can be more pronounced than the neutral drag - the probe drifts in strong fields of neighboring dust particles. Qualitatively, this is taken into account in (26).

The sustained probe velocity $u_{s}=F_{\mathrm{drv}} / \nu_{p} M_{p}$ calculated using Eqs. (20), (21), (24), and (26) is shown in Fig. 4 for two dust particle radii. For this estimation, we

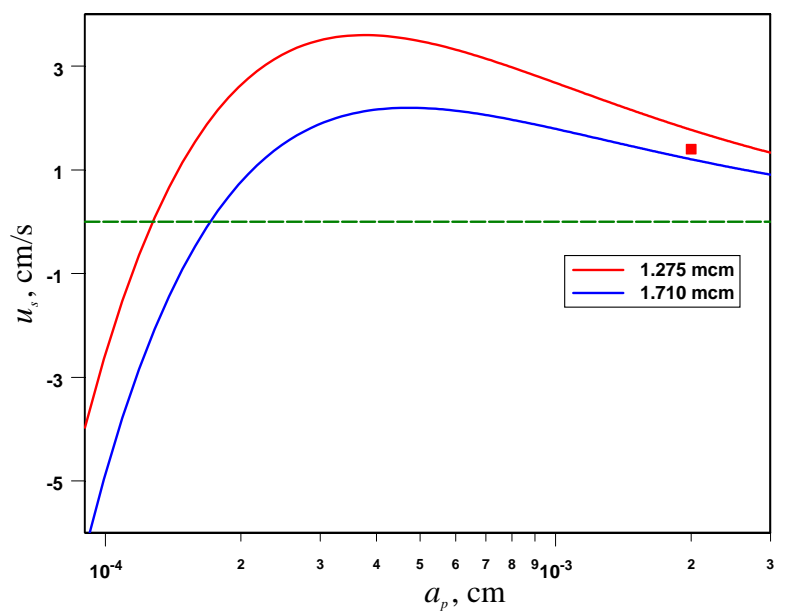

FIG. 4. Probe velocity as a function of its radius under conditions of the experiment ${ }^{38,41}$ for $a=1.275 \times 10^{-4}$ (red line) and $1.71 \times 10^{-4} \mathrm{~cm}$ (blue line). Red dot indicates the probe velocity from the experiment. ${ }^{38,41}$

used the dust particle number density profiles determined recently for the foot region of the dust clouds. ${ }^{42}$ For these profiles, $\ln n_{d} / d y \approx-1.65 \mathrm{~cm}^{-1}$ for $a=1.275 \times 10^{-4} \mathrm{~cm}$ and $\ln n_{d} / d y \approx-1.87 \mathrm{~cm}^{-1}$ for $a=1.71 \times 10^{-4} \mathrm{~cm}$ (processing these data assumed that $\beta=1$ ), see Figs. 5 and 6 of Ref. 42, respectively. The electron temperature amounts to $3.5 \mathrm{eV}$. As $a_{p}$ is increased, the probe velocity changes its sign at $a_{p}=a$. It can be readily deduced from (21) and (26) that $u_{s} \propto 1 / a_{p}$ at $a_{p} \rightarrow \infty$. Hence, $u_{s}\left(a_{p}\right)$ has a maximum whose location depends on $a$ (Fig. 4). In the experiment, ${ }^{38,41}$ the probe radius was assumed to be $a_{p}=7.5 \times 10^{-4} \mathrm{~cm}$ and its velocity reached $1.4 \mathrm{~cm} / \mathrm{s}$, which is of the same order of magnitude as in Fig. $4\left(u_{s} \simeq 3.0 \mathrm{~cm} / \mathrm{s}\right)$. Note that the radius of a probe particle could be underestimated in Ref. 38. Thus, at $a_{p}=2.0 \times 10^{-3} \mathrm{~cm}, u_{s} \simeq 1.77 \mathrm{~cm} / \mathrm{s}$. Note that for this probe radius, the velocity relaxation time $\nu_{p}^{-1} \simeq 0.3 \mathrm{~s}$ is close to the total time of probe motion in a dust cloud $(0.4 \mathrm{~s})$, during which the sustained velocity cannot be attained, i.e., the effect of inertia can be essential. The difference between the theoretical estimate and experiment may also arise from a significant error of Eq. (2) at high ion drift velocity comparable with their thermal velocity, which is characteristic of experiment. ${ }^{41}$ Another source of error could be the neglect of the mechanisms of particle charging other than the OML approximation such as the ion-neutral collisions. ${ }^{43,44}$ It was demonstrated that neglect of the collision effect overestimates somewhat the particle charge but this effect is small at the gas pressure less than $30 \mathrm{~Pa}$ and $Z n_{d} / n_{e}>1$. Such conditions are typical for the experiments treated in this work. Note that since both SP and MSP neglect the dependence of $\sigma_{\text {eff }}$ on the ion energy, inclusion of the ion-particle collisions in the calculation of the ion mean free path does 


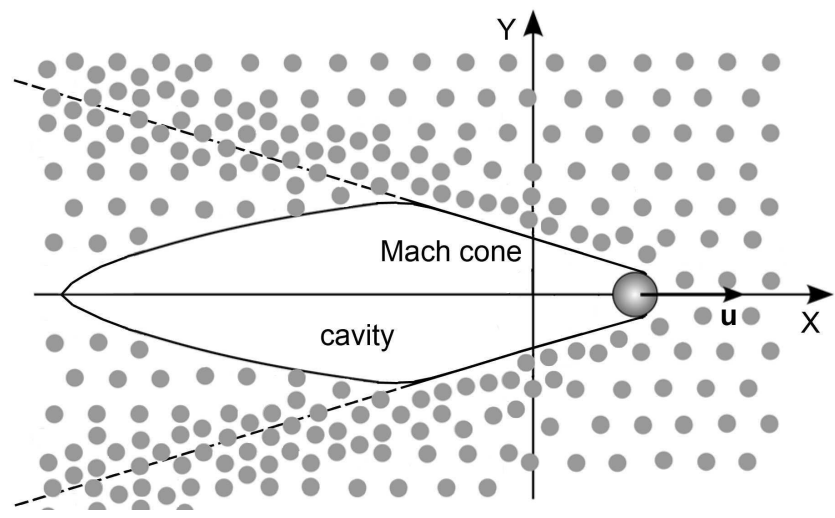

FIG. 5. Sketch showing a subsonic projectile (large bullet point) moving with the velocity $u$ along the $X$-axis in a particle fluid (small bullet points around), with a neutral gas as a still background. The solid line indicates the boundary of a cavity and dashed line, the Mach cone that is the surface of particle number density perturbation.

not change the particle charge. Hence, the allowance for the effect of ion-neutral collisions would lead to an insignificant change of the results.

The discussion above implied that a probe is subsonic and a spherical cavity is formed around it as it moves through the dust cloud. In contrast, for a supersonic probe, a cavity is formed behind rather than around the probe, as it is illustrated with Fig. 5 (see, e.g., Ref. 11). Parameters of such cavity are discussed in the Appendix A. For a very crude estimation, one can assume that in the case of a large probe $\left(a_{p} \gg a\right)$, the ionprobe collision cross section is limited by that for a dust particle. Then $F_{\mathrm{drv}} \simeq\left(Z_{p}-Z\right) e E \simeq Z_{p} T_{e}\left(d \ln n_{e} / d y\right)$ almost coincides with the electric force. Since typically $d \ln n_{e} / d y<0$, a supersonic probe is driven toward the discharge center, i.e., in the opposite direction as compared to a subsonic probe. This effect was observed in experiment, ${ }^{11}$ where the probe velocity varied from 8 to $3.7 \mathrm{~cm} / \mathrm{s}$.

\section{CONCLUSION}

In this work, we have calculated the sum of the ion drag force and the electric force (driving force) acting on a probe particle that finds itself in a dust cloud formed by the particles of the radius different from that of a probe in complex plasma of the low-pressure radio frequency discharge under microgravity conditions. We have modified our approach developed recently to include the effect of the ion collisions against the particles forming the cloud. This effect results in shortening the ion mean free path and, consequently, in the decrease of the moment transfer from the ions to the dust particles, i.e., to the decrease of the ion drag force. However, we have shown that the modified IEOS can be written in the form proposed in the early study. ${ }^{35}$ At the same time, the modified IEOS extends the range of plasma parameters considerably. Thus, it shifts the IEOS critical point toward much higher number densities of the ions, electrons, and particles. Fortunately, the velocity of dust acoustic waves calculated with the modified IEOS has a minor difference from that calculated with the initial version of IEOS and therefore, it still matches the experimental data.

In contrast to our early model (SP), in which the driving force vanishes, the modified model (MSP) yields a nonzero force, whose direction is uniquely defined by the ratio between the radius of the probe and of the dust particle. Namely, if the probe is smaller than a dust particle then the probe moves toward the discharge center against the ion flux, and the large probe moves in the same direction as the ions. Hence, obtained results provide an interpretation for the regularities observed in experiments where subsonic probes were studied. Estimation of the probe typical velocity leads to a reasonable correspondence with available experimental data. The developed model and the driving force calculated on its basis can serve as the ansatz for future investigation of such nonequilibrium phase transition in threedimensional complex plasmas as the lane formation.

\section{ACKNOWLEDGMENTS}

This research is supported by the Russian Science Foundation Grant No. 14-50-00124.

\section{Appendix A: PARAMETERS OF A CAVITY BEHIND THE SUPERSONIC PROBE}

We will estimate the maximum cavity width and the length of a cavity behind a supersonic probe moving with the velocity $u$. If $u \gg c_{s}$ then the Mach cone with contact discontinuity at its surface is realized (Fig. 5). If the probe is large, $M_{d} \ll M_{p}$, the velocity modulus of the dust particle scattered on the probe preserves. In this case, the transversal velocity component of the scattered dust particle is $u_{\perp}=u \sin \alpha=$ $u / M=c_{s}$, where $\alpha$ is the Mach angle and $M=u / c_{s}$ is the Mach number. The expansion time is $1 / \nu$, where $\nu=(8 \sqrt{2 \pi} / 3)\left(\delta m_{n} n_{n} v_{T} a^{2} / M_{d}\right)$ is the friction coefficient for a dust particle. Hence, the transversal dust particle displacement that coincides with the maximum cavity width is $\rho=c_{s} / \nu$. The Mach cone expansion length is then $l_{e}=\rho / \tan \alpha \simeq \rho M=u / \nu$.

Closing of the cavity is defined by the particle number density relaxation. Equation (30) in Ref. 35 is appropriate for the evolution of a density perturbation in complex plasmas. For a low-frequency perturbation, the overdamped regime takes place, and this equation as- 
sumes the form

$$
\frac{\partial \psi}{\partial t}=\frac{c_{s}^{2}}{\nu} \Delta \psi
$$

where $\mathbf{v}=\boldsymbol{\nabla} \psi$ is the local dust particle velocity. It follows from (A1) that the closing time and the corresponding closing length are $\tau_{c}=\nu \rho^{2} / c_{s}^{2}$ and $l_{c}=\nu M \rho^{2} / c_{s}$, respectively. The total cavity length $l=l_{e}+l_{c}$ is then

$$
l=2 \rho M=\frac{2 u}{\nu}
$$

Note that within the accuracy of these estimates, $l_{e}$ proves to be equal to $l_{c}$. Since the damping frequency of dust acoustic waves is $\nu / 2,{ }^{35}$ the length of Mach cone axis, $2 u / \nu$, must coincide with the total cavity length (A2).

Due to a strong decrease of the Coulomb momentum transfer cross section, an ultrafast probe can move through a dust cloud almost without perturbation of the cloud, i.e., without a cavity behind it. The threshold velocity of a probe can be estimated if we assume that the transversal particle displacement is equal to $r_{d} / 2$. Obviously, the typical impact parameter is the same, $\rho_{0} \simeq r_{d} / 2$. For the transversal direction, the dust particle equation of motion has the form

$$
\ddot{z}+\nu \dot{z}=\frac{a a_{p} \Phi^{2} T_{e}^{2}}{M_{d} e^{2}} \frac{1}{u t+\rho_{0}^{2}} .
$$

At the first stage, the particle gains momentum from a probe, $|\ddot{z}| \gg \nu|\dot{z}|$, and its displacement is small. At the second stage, the particle is decelerated due to the neutral drag, $\ddot{z}+\nu \dot{z} \simeq 0$. Calculation of the transversal displacement makes it possible to obtain the estimate for threshold velocity

$$
u_{\mathrm{th}}=\frac{8 a a_{p} \Phi^{2} T_{e}^{2}}{\nu M_{d} e^{2} r_{d}^{2}} .
$$

For $u>u_{\text {th }}$, the cavity does not open.

We will estimate the calculated parameters of a cavity behind a supersonic probe for the experiment, ${ }^{2}$ where $a=4.775 \times 10^{-4} \mathrm{~cm}, M_{d}=6.89 \times 10^{-10} \mathrm{~g}, r_{d}=$ $2.29 \times 10^{-2} \mathrm{~cm}, T_{e}=4.5 \mathrm{eV}, \Phi=1.8, c_{s}=2.0 \mathrm{~cm} / \mathrm{s}$, $\nu=33.5 \mathrm{~s}^{-1}, a_{p}=10^{-3} \mathrm{~cm}$, and $u=13.2 \mathrm{~cm} / \mathrm{s}$ $(\mathrm{M}=6.6)$. Under these conditions, we obtain the maximum cavity width $\rho \simeq 0.06 \mathrm{~cm}$, its expansion velocity $u_{\perp}=2.0 \mathrm{~cm} / \mathrm{s}$, and its total length $l \simeq 0.79 \mathrm{~cm}$ (Eq. (A2)). The experimental parameters are $0.05 \mathrm{~cm}$, $2.1 \mathrm{~cm} / \mathrm{s}$, and $1.1 \mathrm{~cm}$, respectively. One can testify a satisfactory correspondence between these estimates and the experiment. From (A4), we obtain the threshold velocity $u_{\mathrm{th}}=232 \mathrm{~cm} / \mathrm{s}$, which also correlates with the experimental assessment $\left(u_{\mathrm{th}}=158 \mathrm{~cm} / \mathrm{s}\right)$. Consider the experiment, ${ }^{11}$ for which $\nu=49 \mathrm{~s}^{-1}, c_{s}=2.8 \mathrm{~cm} / \mathrm{s}$, and $u=6 \mathrm{~cm} / \mathrm{s}$. Our estimations yield $\rho \simeq 0.057 \mathrm{~cm}$ and $l \simeq 0.25 \mathrm{~cm}$ vs. $0.045 \mathrm{~cm}$ and $0.31 \mathrm{~cm}$ from the experiment.
* dmr@ihed.ras.ru; http://oivtran.ru/dmr/

1 G. E. Morfill, U. Konopka, M. Kretschmer, M. RubinZuzic, H. M. Thomas, S. K. Zhdanov, and V. Tsytovich, New J. Phys. 8, 7 (2006).

2 D. Caliebe, O. Arp, and A. Piel, Phys. Plasmas 18, 073702 (2011).

3 A. Piel, O. Arp, M. Klindworth, and A. Melzer, Phys. Rev. E 77, 026407 (2008).

${ }^{4}$ K. O. Menzel, O. Arp, and A. Piel, Phys. Rev. E 83, 016402 (2011).

5 O. Arp, D. Caliebe, and A. Piel, Phys. Rev. E 83, 066404 (2011).

6 M. Schwabe, S. K. Zhdanov, H. M. Thomas, A. V. Ivlev, M. Rubin-Zuzic, G. E. Morfill, V. I. Molotkov, A. M. Lipaev, V. E. Fortov, and T. Reiter, New J. Phys. 10, 033037 (2008).

7 G. E. Morfill, H. M. Thomas, U. Konopka, H. Rothermel, M. Zuzic, A. Ivlev, and J. Goree, Phys. Rev. Lett. 83, 1598 (1999).

8 S. A. Khrapak, B. A. Klumov, P. Huber, V. I. Molotkov, A. M. Lipaev, V. N. Naumkin, H. M. Thomas, A. V. Ivlev, G. E. Morfill, O. F. Petrov, V. E. Fortov, Yu. Malentschenko, and S. Volkov, Phys. Rev. Lett. 106, 205001 (2011).

9 H. M. Thomas, G. E. Morfill, V. E. Fortov, A. V. Ivlev, V. I. Molotkov, A. M. Lipaev, T. Hagl, H. Rothermel, S. A. Khrapak, R. K. Suetterlin, M. Rubin-Zuzic, O. F. Petrov, V. I. Tokarev, and S. K. Krikalev,
New J. Phys. 10, 033036 (2008).

10 K. Jiang, V. Nosenko, Y. F. Li, M. Schwabe, U. Konopka, A. V. Ivlev, V. E. Fortov, V. I. Molotkov, A. M. Lipaev, O. F. Petrov, M. V. Turin, H. M. Thomas, and G. E. Morfill, Europhys. Lett. 85, 45002 (2009).

11 M. Schwabe, K. Jiang, S. Zhdanov, T. Hagl, P. Huber, A. V. Ivlev, A. M. Lipaev, V. I. Molotkov, V. N. Naumkin, K. R. Sütterlin, H. M. Thomas, V. E. Fortov, G. E. Morfill, A. Skvortsov, and S. Volkov, Europhys. Lett. 96, 55001 (2011).

12 V. E. Fortov and G. E. Morfill, eds., Complex and Dusty Plasmas: From Laboratory to Space, Series in Plasma Physics (CRC Press, Boca Raton, FL, 2010).

13 J. H. Chu and Lin I, Phys. Rev. Lett. 72, 4009 (1994).

14 H. Thomas, G. E. Morfill, V. Demmel, J. Goree, B. Feuerbacher, and D. Möhlmann, Phys. Rev. Lett. 73, 652 (1994).

15 Y. Hayashi and S. Tashibana, Jpn. J. Appl. Phys. 33, L804 (1994).

16 S. V. Vladimirov, K. Ostrikov, and A. A. Samarian, Physics and Applications of Complex Plasmas (Imperial College, London, 2005).

17 V. Fortov, A. Ivlev, S. Khrapak, A. Khrapak, and G. Morfill, Phys. Rep. 421, 1 (2005).

$18 \mathrm{P}$ K. Shukla and B. Eliasson, Rev. Mod. Phys. 81, 25 (2009).

19 M. Bonitz, C. Henning, and D. Block, Rep. Prog. Phys. 73, 066501 (2010). 
20 B. Schmittmann and R. Zia, Phys. Rep. 301, 45 (1998).

21 M. Ciamarra, A. Coniglio, and M. Nicodemi, Phys. Rev. Lett. 97, 038001 (2006).

${ }^{22}$ R. R. Netz, Europhys. Lett. 63, 616 (2003).

23 D. Helbing, I. J. Farkas, and T. Vicsek, Phys. Rev. Lett. 84, 1240 (2000).

${ }^{24} \mathrm{H}$. Löwen and J. Dzubiella, Faraday Discuss. 123, 99 (2003).

25 J. Dzubiella, G. P. Hoffmann, and H. Löwen, Phys. Rev. E 65, 021402 (2002).

26 J. Dzubiella and J. Phys.: Condens. Matter 14, 9383 (2002).

27 J. Chakrabarti, J. Dzubiella, Europhys. Lett. 61, 415 (2003).

28 J. Chakrabarti, J. Dzubiella, and H. Löwen, Phys. Rev. E 70, 012401 (2004).

29 M. E. Leunissen, C. G. Christova, A.-P. Hynninen, C. P. Royall, A. I. Campbell, A. Imhof, M. Dijkstra, R. van Roij, and A. van Blaaderen, Nature 437, 235 (2005).

30 A. Wysocki and H. Löwen, J. Phys.: Condens. Matter 16, 7209 (2004).

31 A. Wysocki and H. Löwen, Phys. Rev. E 79, 041408 (2009).

32 K. R. Sütterlin, A. Wysocki, A. V. Ivlev, C. Räth, H. M. Thomas, M. Rubin-Zuzic, W. J. Goedheer, V. E. Fortov, A. M. Lipaev, V. I. Molotkov, O. F. Petrov, G. E. Morfill, and H. Löwen, Phys. Rev. Lett. 102, 085003 (2009).

${ }^{33}$ G. E. Morfill, A. V. Ivlev, and H. M. Thomas,
Phys. Plasmas 19, 055402 (2012).

34 D. I. Zhukhovitskii, V. I. Molotkov, and V. E. Fortov, Phys. Plasmas 21, 063701 (2014).

35 D. I. Zhukhovitskii, Phys. Rev. E 92, 023108 (2015).

36 H. M. Mott-Smith and I. Langmuir, Phys. Rev. 28, 727 (1926).

37 J. E. Allen, Phys. Scr. 45, 497 (1992).

38 D. I. Zhukhovitskii, V. E. Fortov, V. I. Molotkov, A. M. Lipaev, V. N. Naumkin, H. M. Thomas, A. V. Ivlev, M. Schwabe, and G. E. Morfill, Phys. Rev. E 86, 016401 (2012).

39 P. Epstein, Phys. Rev. 23, 710 (1924).

40 A. V. Ivlev and D. I. Zhukhovitskii, Phys. Plasmas 19, 093703 (2012).

41 D. I. Zhukhovitskii, A. V. Ivlev, V. E. Fortov, and G. E. Morfill, Phys. Rev. E 87, 063108 (2013).

${ }^{42}$ V. N. Naumkin, D. I. Zhukhovitskii, V. I. Molotkov, A. M. Lipaev, V. E. Fortov, H. M. Thomas, P. Huber, and G. E. Morfill, Phys. Rev. E 94, 033204 (2016).

43 S. A. Khrapak, S. V. Ratynskaia, A. V. Zobnin, A. D. Usachev, V. V. Yaroshenko, M. H. Thoma, M. Kretschmer, H. Höfner, G. E. Morfill, O. F. Petrov, and V. E. Fortov, Phys. Rev. E 72, 016406 (2005).

44 S. A. Khrapak, B. A. Klumov, P. Huber, V. I. Molotkov, A. M. Lipaev, V. N. Naumkin, A. V. Ivlev, H. M. Thomas, M. Schwabe, G. E. Morfill, O. F. Petrov, V. E. Fortov, Y. Malentschenko, and S. Volkov, Phys. Rev. E 85, 066407 (2012). 